\section{Clinical and scientific letters}

\section{Letters not directly related to articles} published in Clinical Medicine and presenting unpublished original data should be submitted for publication in this section. Clinical and scientific letters should not exceed 500 words and may include one table and up to five references.

Acute hypercapnic respiratory failure (AHRF): looking at long-term mortality, prescription of long-term oxygen therapy and chronic non-invasive ventilation (NIV)

\section{Background}

Noninvasive ventilation (NIV) improves immediate outcomes in patients presenting with acute hypercapnic respiratory failure (AHRF). A previous national audit ${ }^{1}$ has investigated NIV outcomes in AHRF patients with acute exacerbation of chronic obstructive pulmonary disease (COPD) over a 3-month period. But, a more protracted analysis of these patients is needed to determine long-term gains and fatalities. Also, although British Thoracic Society (BTS) guidelines recommend assessment for long-term oxygen therapy (LTOT) and need for chronic NIV after an episode of AHRF, this practice is variable.

\section{Objectives}

Our primary aim was to look at four-year data on mortality following an episode of AHRF. Our secondary aim was to look at the prescription of LTOT and chronic NIV at the time of discharge in the same group.

\section{Methods}

We retrospectively collected data from our NIV database on patients needing NIV for AHRF, in the year ending 31 December 2007. We excluded patients who were established on domiciliary NIV. The follow-up data were collected up until 31 December 2010. Data analysis was done using Microsoft Excel and VassarStats.

\section{Results}

106 patients $($ male $=44 \%$, female $=56 \%)$ were admitted with AHRF. Mean age was 72.1 years (range 31-93 years). 98\% $(\mathrm{n}=104)$ were managed with NIV and only two patients needed intubation. 30\% $(n=32)$ patients had at least two comorbidities, the most common being ischaemic heart disease $(29 \%, \mathrm{n}=31)$ and congestive cardiac failure $(19 \%, \mathrm{n}=21)$.

All-cause mortality was $57 \%(n=60)$, of which 53\% $(n=32)$ died during admission. Median time to death during admission was 7 days (IQ range: 3-16 days) and postdischarge 315 days (IQ range: 43-408 days). Of those discharged $(n=74), 28 \%$ $(\mathrm{n}=21)$ received LTOT, $7 \%(\mathrm{n}=5)$ LTOT with chronic NIV and one chronic NIV alone. Patients with persistent hypoxia had LTOT assessment; however patients with the most severe hypoxia that were unable to tolerate the cessation of oxygen were prescribed LTOT on discharge. There was a trend towards fewer deaths (median time to death 703 days; IQ: 223-1119 days) in severely hypoxic LTOT group $(27 \%, \mathrm{n}=7)$ compared to those discharged without LTOT $(44 \%, \mathrm{n}=21)$ (Odds ratio 1.02 (95\%CI 0.79-1.32)).

\section{Conclusion}

Data show: 1) $57 \%$ mortality at four years and of this more than half died during their admission. Hence, although NIV has been shown to improve outcomes in a select group of patients with AHRF; in an acute hospital setting short term and long-term outcomes are very different, indicating that admission with AHRF carries a poor prognosis. 2) LTOT following an AHRF may provide a survival advantage; therefore, further research is required to determine if the outcome of patients with persistent hypoxia following treatment for AHRF is improved with LTOT at the time of discharge compared to the current recommendations of performing LTOT assessment post discharge.
NAWAID AHMAD

Specialist registrar, respiratory medicine

ANIKA TAITHONGCHAI

Foundation year 1

REHANA SADIQ

Foundation year 1

NAVEED MUSTFA

Consultant respiratory medicine

City General Hospital, Stoke-on-Trent

\section{Reference}

1 Roberts CM, Stone RA, Buckingham RJ et al. Acidosis, non-invasive ventilation and mortality in hospitalized COPD exacerbations. Thorax 2011;66:43-8.

European School of Internal Medicine summer meeting: September 2011

The European Federation of Internal Medicine (EFIM; www.efim.org) was founded in 1996 with the intention of fostering links between European doctors and national societies, including the Royal College of Physicians (RCP). The EFIM has general internal medicine at its heart and holds regular scientific meetings and conferences covering a broad range of topics relevant to the general physician.

A year after the conception of EFIM, the European School for Internal Medicine (ESIM) was born. ESIM was established to enable trainee physicians across the 35 member countries of the EFIM to meet. School meetings are held annually in winter and summer.

In 2011, the School meeting was held in Brighton at the Brighton and Sussex Medical School under the direction of Chris Davidson who, for the third year running, organised a varied and stimulating mixture of lectures, discussions and case presentations (www.esim2011. org).

Ramon Pujol, president of EFIM, gave the opening lecture. He discussed the history of internal medicine and highlighted the differences in the provision of healthcare throughout Europe; for example, in some Mediterranean countries (eg France, Spain and Portugal) general internal medicine is thriving, whereas in others, such as 
Denmark, it is almost nonexistent and all secondary healthcare is specialised. Each country presented an interesting case, ranging from the everyday to the decidedly small print. The case of hantavirus in Finland causing renal failure had us all stumped until the last slide.

The final day was spent at the RCP, where Mervyn Singer delivered the keynote lecture on 'Coping with critical illness', followed much later by renditions of various national anthems. The week was a perfect blend of medicine and strengthening of international relations.

The next ESIM summer school will be held in Turkey in September 2012.

\section{CATRIONA MAYBURY}

JULIE GALSGAARD

Denmark

Clinical coding for electrophysiology and device procedures: why and how to get it right

\section{Introduction}

Clinical and nonclinical codes are used to generate nationally agreed tariffs that are charged to the primary care trust for every patient intervention. It is essential that coding is accurate otherwise the provider (secondary or tertiary) might not be reimbursed appropriately. ${ }^{1}$ Anecdotal evidence suggested that coding teams were finding it difficult to code electrophysiology (EP) and device procedures correctly because of case complexity. Therefore, we hypothesised that clinical coding for EP and device cases was inaccurate and that this had important implications. We undertook a study to identify the problem, devise a solution and complete the audit loop to determine the effectiveness of our strategy. We agreed that the audit standard would be accurate coding in $80 \%$ of cases.

\section{Data collection}

A retrospective single-centre analysis of 98 consecutive EP procedures and 62 device

Table 1. Data demonstrating accuracy of coding and the shortfall in the subsequently generated tariff as a result of inaccurate clinical codes.

\begin{tabular}{lcccc} 
& \multicolumn{2}{c}{ EP cases } & \multicolumn{2}{c}{ Device cases } \\
& Pre-tick sheet & Post-tick sheet & Pre-tick sheet & Post-tick sheet \\
Coding accuracy & $39 \%$ & $80 \%$ & $45 \%$ & $100 \%$ \\
$\begin{array}{l}\text { Shortfall in generated } \\
\text { tariff (/100 cases) }\end{array}$ & $£ 1,951$ & $f 0$ & $£ 30,919$ & $£ 0$
\end{tabular}

cases was undertaken over a 5-month period. A single clinician, blinded to the previously assigned codes, recoded each case appropriately and used national software to generate a corrected tariff to compare with the original tariff assigned by the coder.

\section{Results}

Overall, there were large inaccuracies in clinic coding (Table 1), particularly in EP cases using mapping systems $(<12 \%$ correct) and complex device implants $(<25 \%$ correct). For EP procedures, this did not greatly influence the overall tariff, principally because $60 \%$ of atrial fibrillation ablations were coded as atrial flutter; the latter generating a higher tariff. For devices, there was a significant shortfall in generated tariff as a result of inaccurate coding

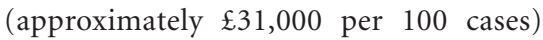
driven largely by complex devices (eg implantable defibrillators and resynchronising pacemakers) being miscoded as simple devices. These data confirmed our hypothesis and showed that coding accuracy was well below the acceptable standard. There were two problems; the clinical procedural details were not compatible with the description in the coding manual of the intervention and the coding manual was also ambiguous, with subtle statements reflecting important differences in procedural detail. For example, Code K575 ( ...percutaneous transluminal ablation of atrial wall') is the correct code for atrial tachycardia ablation, rather than for atrial fibrillation ablation (K621) or flutter ablation (K622), which themselves have similarly ambiguous code descriptors.

\section{Intervention}

The clinicians and coders collaborated on the production of a simple tick-sheet that was an interface between the clinical procedure, coding manual description of the intervention and clinical code. The clinician would complete this simple pro-forma at the end of the case, which would immediately assign a case code and support the coder's task. Six months later, a re-audit was undertaken

\section{Results of the re-audit}

Data from 52 consecutive EP procedures and 24 device cases were analysed. As a result of the tick-sheet, $80 \%$ of EP procedures and $100 \%$ of device cases were now coded correctly, with marked improvement (elimination) of any shortfall in the subsequently generated tariff (Table 1). The set standards were met and exceeded.

\section{Conclusions}

This audit demonstrates how a multidisciplinary approach to clinical coding led to a simple yet powerful tool to improve accuracy and so ensure correct reimbursement.

ANDREW HOGARTH Specialist registrar

MUZAHIR TAYEBJEE Consultant

LEE GRAHAM

Consultant

CHRIS PEPPER

Consultant

Department of Cardiology, The Yorkshire Heart Centre, Leeds General Infirmary

\section{Reference}

1 Department of Health, 2011. Payment by results. www.dh.gov.uk/health/category/ policy-areas/nhs/resources-for-managers/ payment-by-results/ [Accessed 2 February 2012]. 